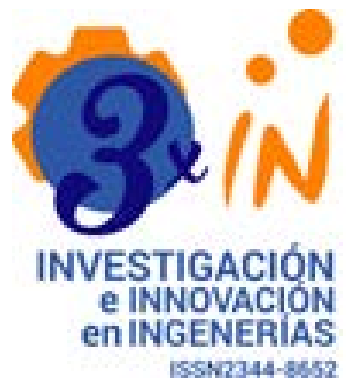

Open Access:

Recibido:

10 agosto de 2017

Aceptado:

5 diciembre de 2017

Correspondencia:

carrietagu@uninpahu.edu.co

ddiazto@uninpahu.edu.co

Jgarzon11@uninpahu.edu.co

cdonosoal@uninpahu.edu.co

DOI

10.17081/invinno.6.2.3111

\section{() $\mathrm{C}$}

Copyright: Arrieta et al

\section{El software como optimizador de oferta y demanda en el sector textil}

\author{
The software as an optimizer of supply and \\ demand in the textile sector
}

\author{
Cristian Andrés Arrieta Gutiérrez, Dany Ferney Díaz Torrez, \\ Jhon Alejandro Garzón Vinasco, Carlos Gilberto Donoso \\ Fundación Universitaria Uninpahu, Colombia
}

\section{Resumen}

Objetivo: Consultar el estado de gestión en las cadenas de suministro del sector textil, para reconocer las inconsistencias de los procesos de oferta/demanda, buscando la manera de optimizar.

Metodología: Como foco de estudio se consideró el sector textil colombiano puntualizando el mercado; se estudiaron procesos cruciales de la gestión de demanda/oferta, la investigación evidencio prácticas ineficientes y se reconoció un problema en el control de proyección frente a la demanda, generando errores de planeación, entrega a tiempos desfasados de los presupuestados, inventarios en stocks generando costos adicionales y daños en las mercancías.

Resultados: La propuesta de un desarrollo, libre de licenciamiento orientado a la planificación de recursos empresariales (ERP), permitirá realizar un control administrativo en la oferta/demanda, se optimizara el uso de tiempos, sistematizando procesos de inventario y distribución, mejorando la productividad de la industria y disminuyendo costos de almacenamiento, la investigación evidencio estos procesos como puntos críticos y son sistematizados de manera eficaz por la interfaz modular, el desarrollo apoyara en la gestión de oferta/demanda.

Conclusión: La fortaleza de la implementación del producto se encuentra en un servicio orientado a empresas que puedan acceder a herramientas de control administrativo que mejore el uso efectivo de sus insumos en la gestión de la oferta/demanda.

Palabras clave: Gestión, demanda, almacenamiento, inventario, costos.

\section{Abstract}

Objective: To consult the state of management in the supply chains of the textile sector, to recognize the inconsistencies of the supply / demand processes, looking for ways to optimize. Methodology: The focus of the study was on the Colombian textile sector, highlighting the market; crucial processes of demand / supply management were studied, research evidenced inefficient practices and a projection control problem was recognized in front of the demand, generating planning errors, delivery to outdated times of the budgeted ones, inventories in stocks generating additional costs and damage to the goods.

Results: The proposal of a development, free of licensing oriented to the planning of business resources (ERP), will allow an administrative control in the offer / demand, the use of time will be optimized, systematizing inventory and distribution processes, improving productivity of the industry and decreasing storage costs, the research evidenced these processes as critical points and are systematized effectively by the modular interface, the development will support supply / demand management.

Conclusion: The strength of the implementation of the product is in a service oriented to companies that can access administrative control tools that improve the effective use of their inputs in the supply / demand management.

Keywords: Management, demand, storage, inventory, costs.

Como citar este articulo (IEEE) C. Arrieta, D. Díaz, J. Garzón, y C. Donoso, "El software como optimizador de oferta y demanda en el sector textil", Revista Investigación e Innovción en Ingenierias, vol. 6, n. 2, 2018. DOI: 10.17081/invin$\underline{\text { no.6.2.31111 }}$ 


\section{Introducción}

Esta investigación se centra en la búsqueda de una solución de software que pueda apoyar diferentes necesidades de la cadena de suministro de procesos logísticos en el sector textil, necesidades que dejan atrás una cantidad crítica de empresas a nivel competitivo. Concretamente, se trata de la elaboración de un módulo de libre acceso que sirva para apoyar considerablemente la cadena de suministro de las empresas en las áreas de estrategia, planificación y gestión de oferta-demanda.

El objetivo principal de la investigación consiste, así, en generar las condiciones de desarrollo para que se integre la interface modular que controle la gestión de demanda-oferta, órdenes de pedido y reportes, con los módulos ya desarrollados de gestión de proveedores y gestión de inventarios dentro del Idempiere, de modo que se consoliden los procesos logísticos de la cadena de suministros del sector textil.

En este orden de ideas, es relevante mencionar algunos conceptos previos, que permitan una completa comprensión de la aplicabilidad del producto planteado:

\section{¿Qué es la cadena de suministros o cadena de abas- tecimiento?}

Se denomina cadena de suministros a un conjunto de actividades que comprenden desde el proveedor del servicio, pasando por las materias primas, la producción para obtener un producto y, finalmente, la distribución.

La cadena de suministros incluye diferentes áreas de competencia:

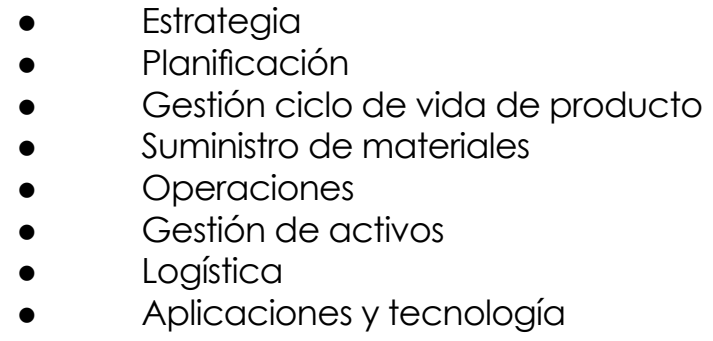

Estas áreas son vitales para la organización ya que constituyen la base del proceso desde la creación del producto hasta su respectiva distribución. Sabido esto. analizaremos enseguida las problemáticas actuales.

La crisis actual de las empresas se debe a circunstancias como: los retrasos de entregas, recorte de precios, crisis en ventas, altos inventarios en stock, mala planeación. Hoy día la cadena de suministro de las empresas tiende a ser más global, lo que aumenta en muchos niveles la competitividad en el mercado. Las empresas deben estar preparadas para impulsar más productos a un mercado en constante crecimiento y para el aumento de la complejidad en la misma, ya que esta involucra cada vez a más compañías.

Según un estudio realizado por David Soto, vicepresidente de IBM, los principales desafíos que tienen las empresas con su cadena de abastecimiento y para contar con una cadena de suministro inteligente son los siguientes:

- $\quad$ Contención de costes

- Visibilidad de la cadena de suministro

- $\quad$ Gestión del riesgo

- $\quad$ Clientes más exigentes

- Globalización

En ese mismo marco, las debilidades que actualmente existen en la cadena 
de suministros son:

- $\quad$ Contención de costes: Los responsables de la cadena de suministro de la empresa consideran la contención de costes como su responsabilidad número uno y se esmeran en minimizarlos.

- $\quad$ Visibilidad de la cadena de suministro: Las empresas no tienen una visibilidad completa de lo que ocurre en la cadena de suministro.

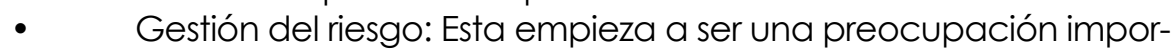
tante, sin embargo no forma parte de la agenda del día a día.

- $\quad$ Clientes más exigentes: La mayoría de las empresas no escuchan al cliente.

- Globalización: La crisis y la globalización empujan a las empresas a comprar y relocalizar más en países de bajo costo.

- Tecnología de la Información: Las empresas requieren herramientas de cálculo de pronósticos que permitan lograr mayor asertividad basada en la demanda o consumo real [1].

De igual forma, cabe resaltar los puntos importantes de una cadena de suministro inteligente, a saber:

- $\quad$ Preparación tecnológica

- Inteligencia

- Interconexión

- $\quad$ Flexibilidad (Costes Variables)

- $\quad$ Agilidad (para responder a cambios de mercado)

lo planteado hasta aquí evidencia que existen unas problemáticas en la cadena de suministros, pero que igualmente hay un ideal de mejora en la práctica [2].

\section{¿Qué es ERP (Enterprise Resources Planning)?}

El ERP es un sistema de información que integra procesos de negocio con el objetivo de crear valor y reducir los costos. Esto se logra al conseguir que la información correcta esté disponible para las personas adecuadas y en el momento adecuado, es decir, en la toma de decisiones relacionadas con la gestiónr de los recursos de manera productiva y proactiva. LOS ERP se componen de varios módulos que sirven y dan apoyo a funciones de la empresa [3].

¿Qué es Open Source (Código abierto)?

Open Source o Código Abierto es el nombre que designa al software distribuido y desarrollado libremente. El código abierto se orienta más a los beneficios prácticos de compartir el código que a las cuestiones éticas y morales que destacan en el llamado software libre [4].

\section{¿Qué es Cross Docking?}

Es un sistema de distribución en el cual la mercancía no se almacena, sino que se prepara inmediatamente para su próximo envío. Es decir, en este sistema no existen stocks ni almacenajes intermedios. Ello "Asegura el movimiento constante de productos, reduciendo el tiempo y los inventarios de toda la cadena del suministro, así se evita al máximo el almacenamiento de los productos" [5].

\section{¿Qué es Open up?}

Es una metodología de desarrollo que asemeja muchos elementos de la metodología RUP, según la wiki de Eclipse se define como: Un proceso unificado de apoyo que se aplica a enfoques iterativos e incrementales dentro de un ciclo de vida estructurado. Open UP abraza una filosofía 
pragmática, ágil, que se centra en la naturaleza colaborativa de desarrollo de software [6].

Es responsable del desarrollo de una parte del sistema, incluyendo el diseño para que se ajuste a la arquitectura, posiblemente prototipando la interfaz de usuario, y luego implementando, generando pruebas unitarias, e integrando los componentes que son parte de la solución [6].

\section{¿Qué es OSGI?}

OSGI es un sistema modular para Java que establece las formas de crear módulos y la manera en que estos deben interactuar entre sí en tiempo de ejecución. OSGl intenta solventar los problemas del tradicional "clases de carga" de la máquina virtual y de los servidores de aplicaciones Java. Este framework proporciona a los desarrolladores un entorno orientado a servicios y basado en componentes, al ofrecer estándares para manejar los ciclos de vida del software.

\section{Método}

Se realiza un estudio de las diferentes prácticas que se puedan aplicar a la investigación y entre ellas cuáles tienen mayor impacto. Como instrumento se utilizaron encuestas para determinar el uso de las tecnologías y el grado en que se comparte información entre los diferentes actores de la cadena.

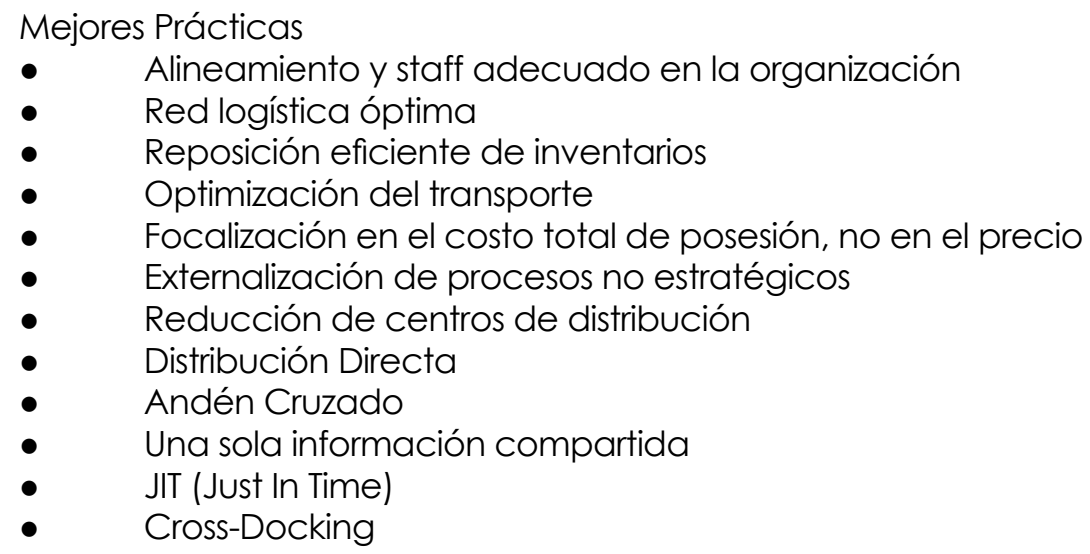

Algunas de estas prácticas quizás sean simples, sencillas y familiares. Otras, nuevas para una compañía. Implementar las que mejor se adapten a un determinado negocio podría ser la base para encontrar la excelencia en su cadena de suministro [7].

La metodología de investigación seleccionada es la anteriormente definida, pues el tipo de problema que se resuelve con la investigación para este producto corresponde en todos los aspectos a los hallazgos tecnológicos encontrados según hipótesis, pero que aún requieren mejoras para su aprovechamiento óptimo, como lo es la herramienta tecnológica ldempiere.

\section{Resultados}

Las encuestas se realizaron a 30 ejecutivos que están directamente relacionados en la cadena de suministros de 6 empresas textiles ubicadas en Bogotá. Se evidenció que un 32\% tenía claro su sistema de gestión de abastecimiento, pero no la capacidad de accesibilidad a las herramientas; en tanto que el $68 \%$ restante aun no tenía conocimiento de los estándares y herramientas de buenas prácticas para la cadena de abastecimiento que 
podrían ayudar a sus procesos logísticos, además de los procesos de oferta-demanda, específicamente con el producto. A continuación se evidencian las preguntas de mayor peso para la investigación.

Figura 1. Tiempo de almacenamiento mercancías

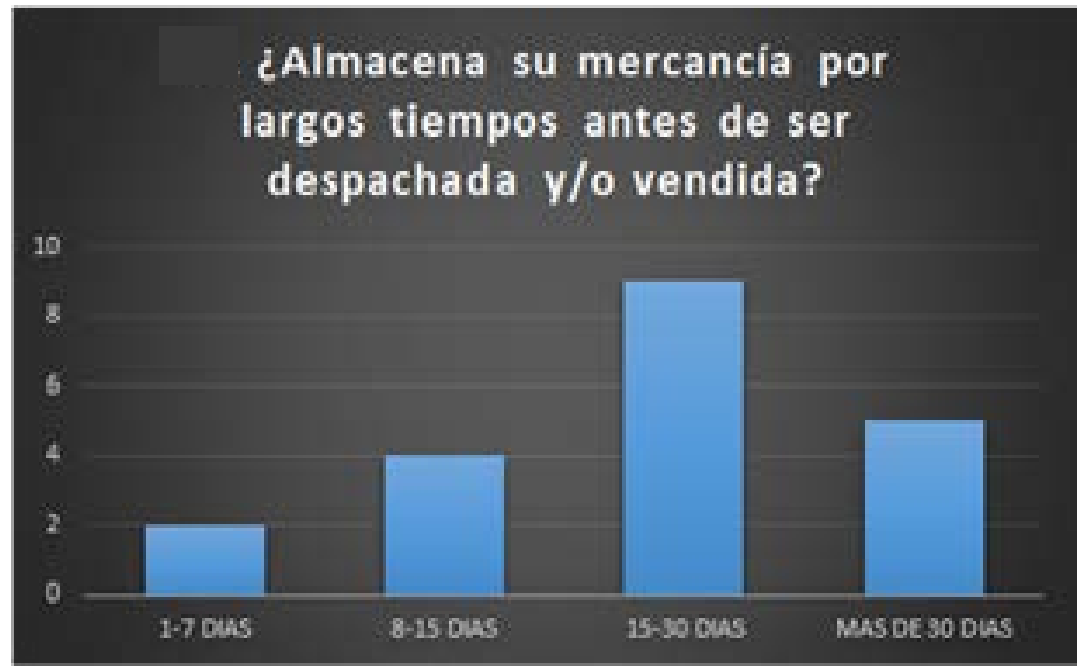

Figura 2. Disponibilidad de las fechas de producto

\section{¿Sus clientes conocen las fechas y \\ los productos que estarán disponibles en estas?}

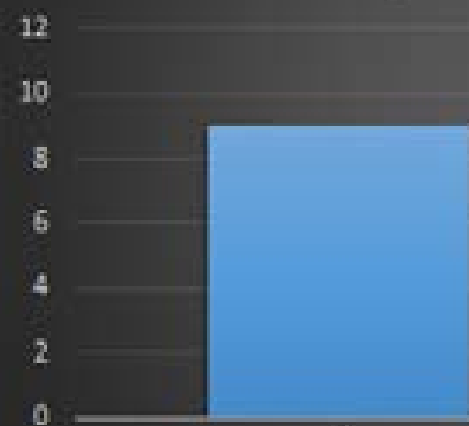

S!
No 
Figura 3. Disponibilidad de las fechas de producto

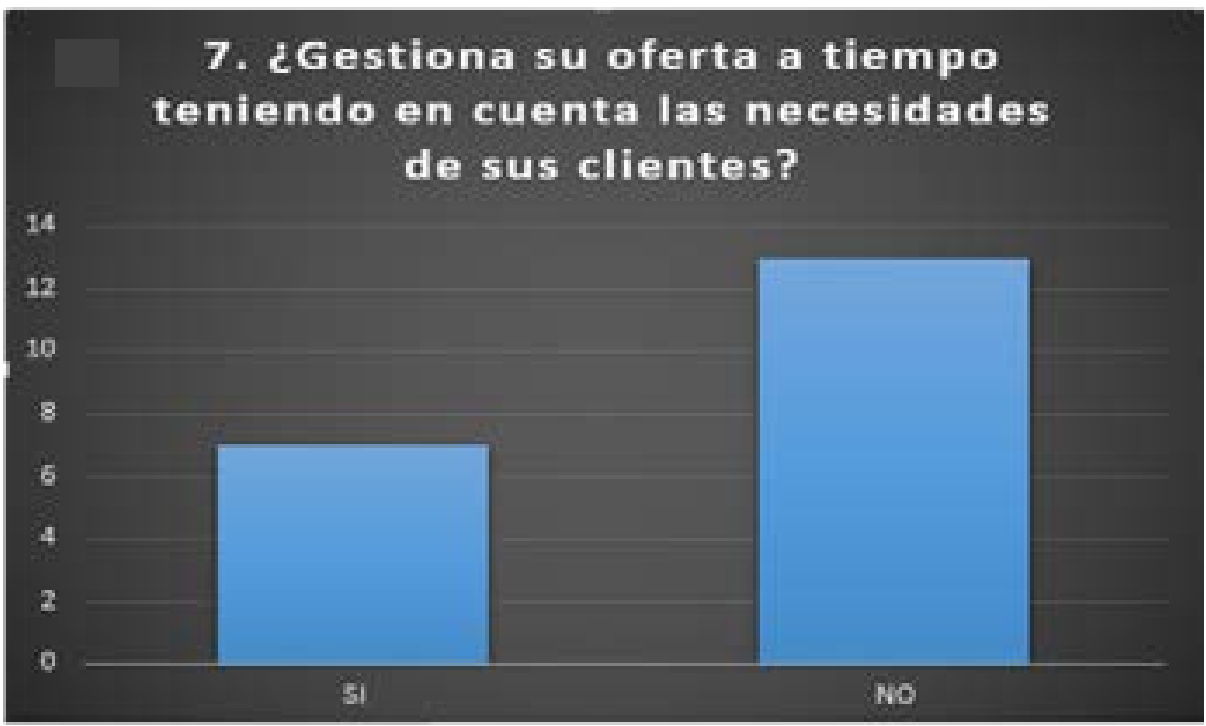

Las gráficas anteriores afianzan el estudio, además de hacer evidente la mínima organización y la intermitencia de la información con los actores de la cadena de abastecimiento de oferta-demanda por parte de las empresas textiles. También se establece que en sus procesos de almacenamiento y distribución no hay un control de calidad que cuente con sus clientes y esto causa la pérdida de un porcentaje de las mercancías y tiempos de reparto, afectando las ganancias y comprometiendo las del cliente. Como trasfondo de las encuestas se reconocen las debilidades ya mencionadas, y se convierten en los nuevos requisitos para mejorar la cadena de suministros dentro de la herramienta Idempiere, mejorada a su vez por una interface modular para la gestión de oferta-demanda.

\section{Análisis del problema}

El problema surge a partir de los estudios realizados por el Clúster de Bogotá y la Cámara de Comercio con relación a la identificación de necesidades logísticas de las industrias. La investigación se inició con los resultados de los talleres, que identificaron la problemática más crítica a fin de darle una solución práctica al problema de las industrias textiles.

Figura 4. Resultados Talleres Clúster

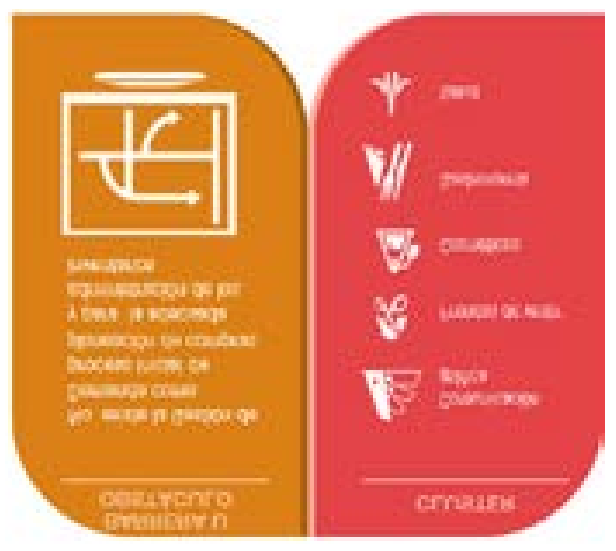

Fuente: [8] 
En los procesos logísticos que presentaban falencias, se detectó que: en los procesos de oferta-demanda, así como en los procesos de stock y en los procesos de distribución había pérdidas innecesarias de tiempo y capital, debido a desinformación de las empresas textiles frente a herramientas tecnológicas que estandarizan los procesos logísticos comerciales.

Al obtener los resultados de las encuestas, se determinó que el proceso con mayor impacto dentro de la logística de las empresas es el de oferta-demanda, el cual no tiene manejo y control de su proyección de mercancía, lo que afecta su stock y su distribución, al igual que el stock del cliente de las empresas de la industria textil.

Figura 5. Cadena de procesos que siguen las industrias sin la herramienta Idempiere

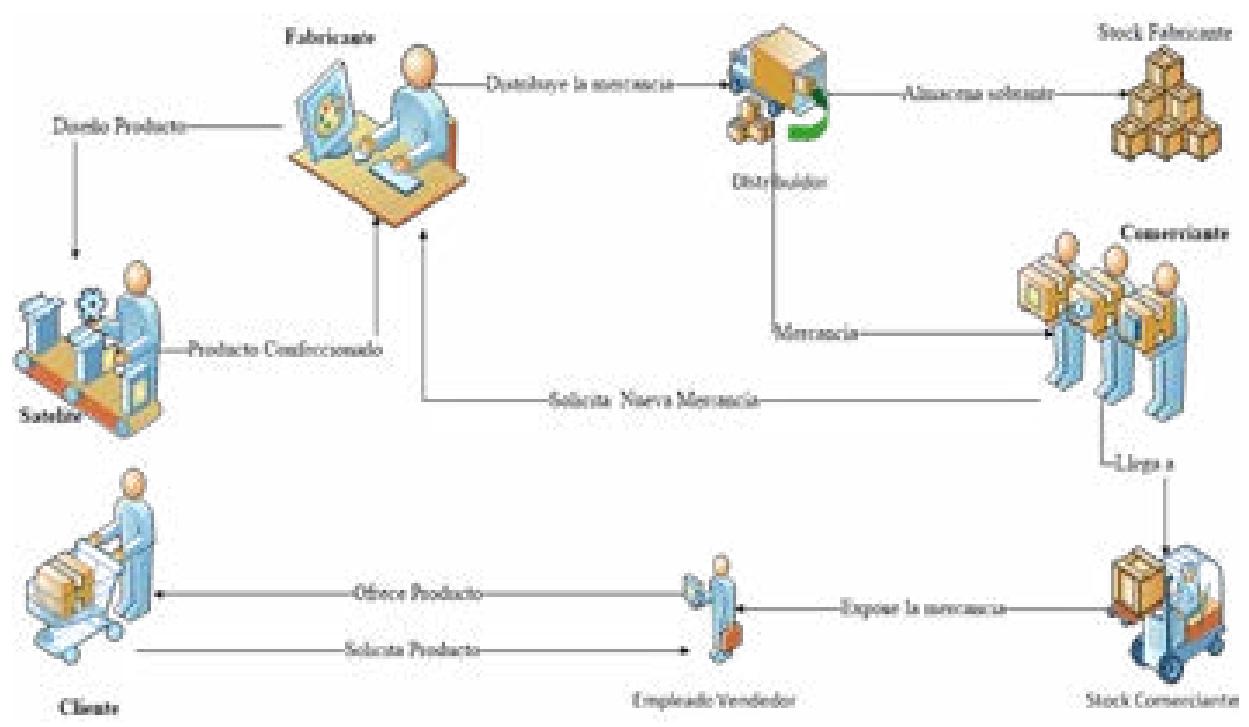

Mediante el planteamiento de la solución para el problema de la investigación, se determinó que los procesos de stock y distribución están fuertemente afectados de manera benéfica, al solucionarse los procesos de gestión de oferta-demanda. Para mejorar este proceso, se creó una herramienta de uso libre y con la capacidad de manejar los procesos logísticos ya existentes y controlados de la industria textil, a excepción de los procesos sobre los cuales la investigación ofrecerá una salida; la herramienta tecnológica a utilizar es Idempiere.

La solución que se ofrece requiere la realización de una interface modular para los procesos de gestión de demanda, donde los usuarios con acceso a la herramienta tecnológica puedan demandar la proyección de los productos a los fabricantes en cantidad y tiempo esperados, De la misma manera estos pueden ofrecer una proyección de sus productos en tiempos específicos; la interface facilita la configuración de proyección acertada a los usuarios. 
Figura 6. Ensamblaje módulos Idempiere con la interface modular MGD

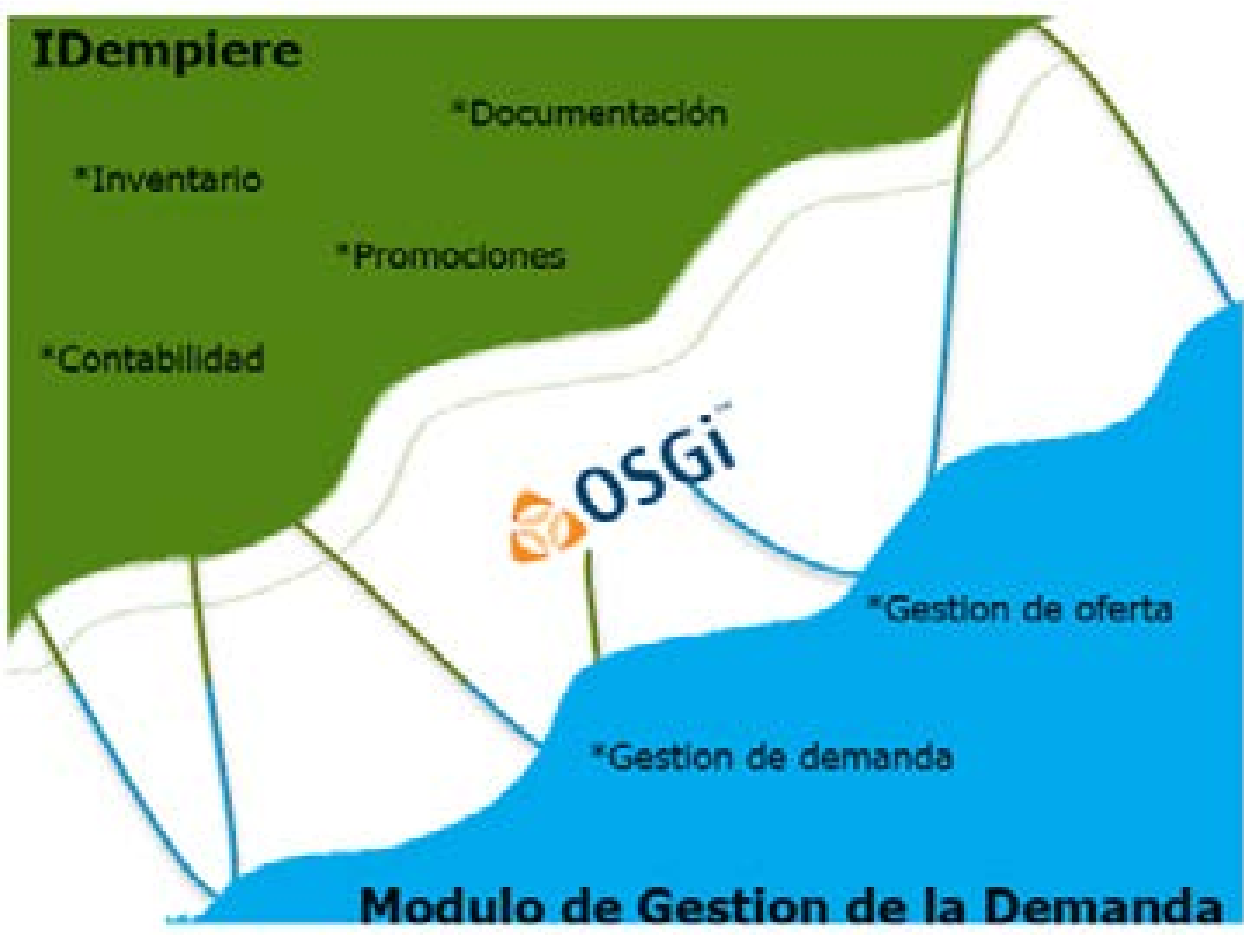

La Figura 6 hace referencia a la integración de Idempiere junto con la interface modular del producto propuesto por la investigación mediante OSGl, la cual es una herramienta que permite diseñar plataformas compatibles. A continuación, se plantea gráficamente la representación del mejoramiento en la cadena de procesos.

Figura 7. Procesos logísticos con el uso de Idempiere y MGD.

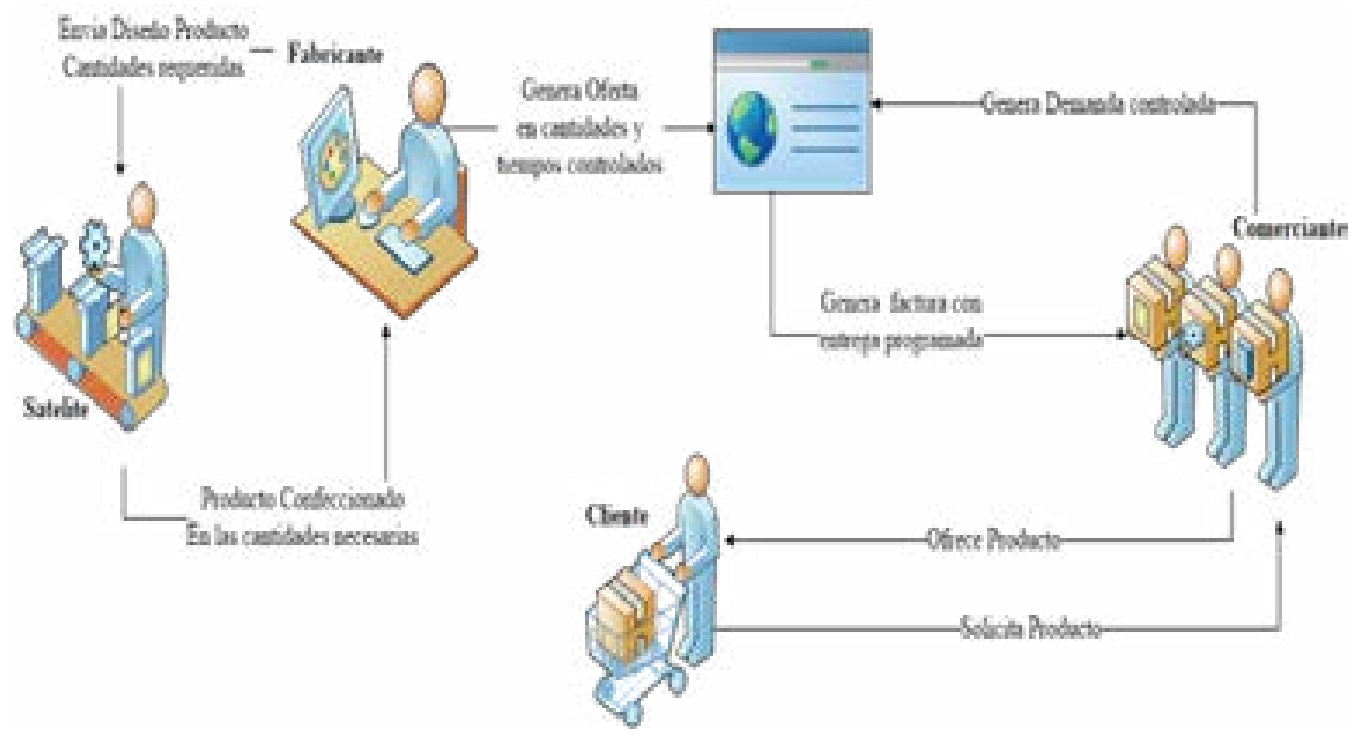




\section{Discusión}

Se encontró una falta de conocimiento o de aplicación correcta en estándares, metodologías y procesos que optimizan la cadena de suministro, tal como se aborda en la investigación realizada por el Clúster de Bogotá y la Cámara de Comercio.Según la investigación realizada se evidencia una falencia en el área de logística en diferentes sectores de la industria; una debilidad encontrada es la falta de conocimiento y visualización de la cadena de suministro por parte de los proveedores y clientes de las etapas o procesos logísticos de la cadena, dejando así en manos de la especulación, información que podría optimizar tiempos y costos. También se encontró que los proveedores solo tienen en cuenta precios o costos, reduciendo los tiempos de búsqueda en largos listados de ofertas mal tipificadas o planteadas. Estos son algunos motivos que podrían afectar seriamente la fidelización de los clientes, lo que refleja un deficiente uso de las TIC que se vuelve relevante en el estudio.

Se demuestra, además, que la gestión de inventarios por medio de cross-docking es nula y que en comparación con la investigación realizada sobre economía aragonesa de Fundear, todavía no se ha adoptado como una práctica habitual. Aun así, se usa en un pequeño porcentaje, como bien se menciona en el documento [9 - 11].

Para terminar se retoma un documento de la universidad de Zaragoza (2006) que afirma que el mejoramiento de la cadena de suministros "supone un claro potencial de desarrollo para las empresas".

\section{Conclusión}

Se puede argumentar que el software que se busca implementar tendrá un impacto benéfico en la reducción de tiempos y disminución de costos en los diferentes procesos logísticos de los fabricantes que intervendrán directamente con la interface modular; de la misma forma, se involucran los procesos desarrollados por los clientes, comerciantes y actores secundarios que intervendrán con el nuevo sistema de procesos, permitiendo que ambos usuarios puedan sincronizar sus actividades procedimentales y recortar algunos insumos hoy en día malgastados por la falta de comunicación asertiva [12].

Con el desarrollo de la interface modular, se reducen los stock (inventarios) del fabricante y el cliente, los cuales generaban pérdida en las mercancías almacenadas por deterioro, demora de circulación del producto. En forma adicional, se mejoran los procesos de distribución ofreciendo una sincronización en los procesos logísticos de ambos actores, lo cual contribuye a la reducción de tiempos de espera para la recepción de la mercancía por parte del cliente.

Como beneficio indirecto de la sistematización de la oferta-demanda se eliminó la tercerización del producto, mejorando tiempos y reduciendo costos de los actores principales de la cadena de abastecimiento.

\section{Referencias bibliográficas}

1. D. Soto, Cadena de suministro inteligente. New York: IBM Corporation, 2008.

2. J. M. Rovira. IBM Global Business Services, la cadena de suministro inteligente del futuro. New York: IBM Corporation, 2008.

3. R. McGaughey y Gunasekaran, Selected Readings on Strategic Information Systems, IGI Global, 2009. 
4. GPS, «gpsopensource,» 2010. [En línea]. Available: https://www.gpsos.es/ soluciones-open-source/definicion-de-open-source/.

5. S. Miquel Peris, F. Parra Guerrero y C. L'hermie, Distribución Comercial, $\mathrm{ESCl}, 1997$.

6. Eclipse, «Open up,» 2012. [En línea]. Available: http://epf.eclipse.org/wikis/openup/.

7. I. Johan Dreher, "Mejores prácticas en la cadena de suministro", Evaluandoerp, p. 1, 2014.

8. Camara de comercio, «Resultado de los Talleres Clusters,» de Talleres Clusters para la Identificación de Necesidades Logísticas, Bogota, 2016.

9. J. Saenz, "supone un claro potencial de desarrollo para las empresas", Aragón, 2006.

10. F. Caballos., J. Betancur Villegas and J. Betancur Villegas, "Simulación

Discreta Aplicada a los Modelos de Atención en Salud", Investigación e Innovación en Ingenierías, vol. 2, no. 2, 2014. DOI: 10.17081/invinno.2.2.2045

11. M. Zurbarán, "Efectos de la Comunicación en una Red Ad-Hoc", Investigacion e Innovación en Ingenierias, vol. 4, no. 1, 2016. DOI: 10.17081/invinno.4.1.202212.

12. H. G. Hernandez Palma, D. Sierra Martinez y D. Cardona, "Enfoque basado en procesos como estrategia de dirección para las empresas de transformación", Saber, Ciencia y Libertas, 11 (1), 2016. 